\title{
Diffusion and applications of musculoskeletal ultrasound in Italian Rheumatology Units
}

\author{
A. lagnocco', F. Ceccarelli', G. Cuomo², A. Delle Sedie ${ }^{3}$, G. Filippou ${ }^{4}$, \\ E. Filippucci ${ }^{5}$, W. Grassi ${ }^{5}$, F. Porta ${ }^{6}$, G. Sakellariou ${ }^{7}$, on behalf of the \\ Musculoskeletal Ultrasound Study Group of the Italian Society of Rheumatology* \\ 'Dipartimento di Medicina Interna e Specialità Mediche, Sapienza Università di Roma; \\ 2Dipartimento di Internistica Clinica e Sperimentale. Seconda Università di Napoli; \\ ${ }^{3}$ U.O. Reumatologia, Azienda Ospedaliero Universitaria Pisana; \\ ${ }^{4}$ Dipartimento di Medicina Clinica e Scienze Immunologiche sezione di Reumatologia, Università di Siena; \\ ${ }^{5}$ Dipartimento di Reumatologia. Università Politecnica delle Marche. Jesi, Ancona; \\ ${ }^{6}$ Dipartimento di Medicina Interna, Università di Firenze; \\ ${ }^{7}$ Unità Operativa di Reumatologia, IRCCS Policlinico S. Matteo, Pavia, Università di Pavia, Italy \\ *Musculoskeletal Ultrasound Study Group of the Italian Society of Rheumatology Members: \\ F. Bandinelli, F. Ceccarelli, O. de Lucia, L. Di Geso, V. Di Sabatino, O. Epis, A. Gabba, A. Gattamelata, \\ M. Gutierrez, L. Massaro, M. Massarotti, C. Perricone, Vi. Ravagnani, L. Riente, C. Scioscia, \\ S. Truglia, C. Venditti
}

Corresponding author:

Annamaria lagnocco

Dipartimento di Medicina Interna

e Specialità Mediche

Sapienza Università di Roma

V.le di Porta Tiburtina, 28 - 00185 Roma, Italy

E-mail: annamaria.iagnocco@uniroma1.it
T1entis he Musculoskeletal Ultrasound Study Group of the Italian Society of Rheumatology (SIR) was founded during the 68 $8^{\text {th }}$ SIR Congress, on November 2011. The request of activation of this group was based on the increasing interest and the widespread diffusion of ultrasound in the scientific rheumatology community and on the solid experience of some Italian rheumatologists in the field. The aims of the Study Group are to stimulate the applications and use of ultrasound in the clinical practice at the level of the Italian rheumatology units and, in addition, to develop research projects at a national level.

In order to know the entity of the diffusion of ultrasound in Italy, a survey has been distributed to the regional delegates as well as to the heads of the rheumatology departments that were mentioned in the SIR web site. Because we didn't have access to the email addresses of all rheumatologists in Italy, and to avoid errors in the transmission of the questionnaire, we decided to use the information reported in the SIR web site. The survey, that was based on a series of questions related to the use of ultrasound by the Italian rheumatologists, was then distributed to the local teams.
The survey consisted of a first short part, that included questions concerning general demographic and professional data, and of a second specific part represented by 14 questions regarding the ultrasonographic training, the applications of ultrasonography in daily clinical practice and the level of ultrasonographic equipment used.

\section{RESULTS}

Sixty-five clinicians (60 rheumatologists and 5 rheumatology's residents), corresponding to the $5.3 \%$ of SIR members, answered the survey.

Thirty-four of them $(52 \%$; 29 rheumatologists and 5 residents) were from 15 different university departments and $30(46 \%)$ from 23 different Italian hospitals. In addition, one survey was completed by a private rheumatologist.

Accordingly, answers were received from $15 / 34(44.1 \%)$ university centers and from 23/61 (37.7\%) Italian hospitals.

The participants in the survey had been working as rheumatologists for 13.5 years on average (range 1-40).

Forty-eight clinicians $(73.8 \%)$ referred to 
perform musculoskeletal ultrasonography as expert sonographers on an average of 7.6 years (range $0.5-22$ ). $77.1 \%$ of them had been trained by attending specific courses on musculoskeletal ultrasonography in rheumatology; $45.8 \%$ had attended either formal or informal training programs provided by experienced rheumatologists or had conducted self-study periods; $20.8 \%$ had attended training projects provided by radiologists; $19.2 \%$ had attended formal ultrasound courses organized by radiologists; $14.6 \%$ had learned ultrasound by following training provided by other specialists and, finally, $6.2 \%$ had used on-line courses. In most cases, more than a single method of approach was used during the training period.

An average of 3 rheumatologists per clinical unit (range 0-8), corresponding to $33 \%$ of the whole local medical team, used to perform musculoskeletal ultrasonography during their daily clinical practice. An average of 2 equipment (range 1-6), with a global number of 67 machines, were available in the different rheumatology units participating in the survey.

Concerning the level of the sonographic equipment that were available at the rheumatology units participating in the project, 28 high-end (41.8\%), 31 medium range (46.3\%), 2 low-end (3\%) and, finally, 6 portable $(8.9 \%)$ machines were used.

An average of 29 scanning sessions per week were performed (range 3-100), and an average of 9.6 hours a week (range 1-30) was spent by the participants in performing musculoskeletal ultrasonography.

Sonography was performed with exclusive clinical purposes in 14 cases $(29.2 \%)$, as a research tool in $2(4.2 \%)$ and aiming at both goals in 32 cases $(66.6 \%)$.

Hands and wrists were the most frequently examined joints $35 / 48(72.9 \%)$, followed by shoulders $10 / 48$ (20.8\%), knees $6 / 48$ $(12.5 \%)$ and lastly by muscular structures of upper and lower limbs, that were evaluated in only one case $(2.1 \%)$.

The most part of ultrasound examinations (34/48 cases; $70.8 \%)$ were performed in order to assess patients with rheumatoid arthritis and other inflammatory conditions.
Ultrasonography was then used to evaluate patients with osteoarthritis in 17/48 cases (35.4\%), with spondyloarthritis in $7 / 48$ $(14.6 \%)$ and with connective tissue diseases in $3 / 48$ cases $(6.2 \%)$.

Finally, musculoskeletal ultrasonography was always used as a guidance for intraor peri-articular procedures (i.e. joint fluid aspirations, injections and biopsies) by 31 ultrasonographers (64.6\%); was applied only occasionally for these purposes by 15 $(31.2 \%)$ of them; $4.2 \%$ of participants had never used ultrasonography for this aim.

\section{CONCLUSIONS}

The survey conducted by the SIR Musculoskeletal Ultrasound Study Group represents the first audit on the diffusion and applications of ultrasonography in the Italian rheumatology units.

This is not the first national survey on ultrasound in rheumatology, being similar studies performed in other countries. Particularly, a national survey addressed to all rheumatology units in Spanish hospitals has been recently published, showing a higher availability of ultrasound equipment $(90 \%)$ and reporting that $38.7 \%$ of Spanish rheumatologists use ultrasound, with half of the units that have at least one rheumatologist especially dedicated to perform it (1).

On the basis of the results obtained and considering the international emerging interest in the field as well as the widespread development of ultrasonography in rheumatology, the Group plans to promote a series of initiatives aiming at improving the applications of musculoskeletal ultrasonography in Italy.

Key words: diffusion, musculoskeletal ultrasound, Italy.

\section{REFERENCES}

1. de Miguel E, Andreu JL, Naredo E, et al. Situation of Spanish echography in Spanish rheumatology 2012. Reumatol Clin. 2012; 8: $310-4$. 